
request.

This paper refers to seven figures (not included). Hard copies of the figures IASSNS-HEP-92/8

MIT-CTP-2067

February 1992

\title{
INTERPOLATING STRING FIELD THEORIES
}

\author{
BARTON ZWIEBACH ${ }^{\star}$ \\ School of Natural Sciences \\ Institute for Advanced Study \\ Olden Lane \\ Princeton, NJ 08540
}

\begin{abstract}
A minimal area problem imposing different length conditions on open and closed curves is shown to define a one parameter family of covariant open-closed quantum string field theories. These interpolate from a recently proposed factorizable open-closed theory up to an extended version of Witten's open string field theory capable of incorporating on shell closed strings. The string diagrams of the latter define a new decomposition of the moduli spaces of Riemann surfaces with punctures and boundaries based on quadratic differentials with both first order and second order poles.
\end{abstract}

$\star$ Permanent address: Center for Theoretical Physics, MIT, Cambridge, Mass. 02139. Supported in part by D.O.E. contract DE-AC02-76ER03069 and NSF grant PHY91-06210. 
A covariant quantum theory of open and closed strings was derived recently from string diagrams defined by a minimal area problem [1]. This problem required that the length of any nontrivial open curve be larger or equal to $l_{o}=\pi$, and that the length of any nontrivial closed curve be larger or equal to $l_{c}=2 \pi$. In the resulting theory one can compute directly all scattering amplitudes involving any possible numbers of open and closed strings on any surface. The Feynman rules of covariant open string theory [2] are simpler, but amplitudes involving external closed strings must be obtained indirectly by factorization. Finding a way of extending the simple string diagrams of open string theory to include external closed strings was the motivation for the present work.

The solution is simple. Open string diagrams are now known to arise from minimal area metrics under the condition that all nontrivial open curves be longer or equal to $\pi[3]$. We can incorporate external closed strings without changing the minimal area problem at all! Closed string external states correspond to punctures inside the surface, and curves cannot be moved across them. Their presence creates new homotopy types of nontrivial open curves. The minimal area metric will have to adjust itself in order to satisfy the new length conditions arising due to the closed string punctures.

The above minimal area problem is recognized to correspond to $l_{o}=\pi$, and $l_{c}=0$. It is therefore natural to consider the generalized problem where $l_{o}=\pi$ and $l_{c}=a$, where $a$ is a constant. We shall see that this defines a one parameter family of string diagrams. Even more interesting is that actually they correspond to a one parameter family of string field theories. This happens simply because the string diagrams can be built by sewing. When $a=2 \pi$ we recover the open-closed factorizable theory [1], and when $a=0$ we get the extended open string theory.

A large part of our effort in this paper will be devoted to elucidate the extended open string theory. Since the minimal area problem is showing us a natural way to incorporate external closed strings into the framework of the open string theory we only have to figure out how the string diagrams look, and if they can be built using Feynman rules. The minimal area metrics we shall find always arise from quadratic differentials, and closed strings appear as first order poles. A first order pole corresponds to a finite area conical singularity of the metric with an angle of $\pi$ at the singularity. We will find the amazingly simple result that a single open-closed interaction is all one needs to add to the Witten open string vertex so 
that the Feynman rules generate the above minimal area string diagrams and thus generate all moduli spaces relevant for open and open-closed string scattering amplitudes. There is no need to add even an open-open-closed vertex. The open-closed vertex appeared first in the factorization studies of Shapiro and Thorn [4] and in the work of Strominger [5]. Its geometry corresponds to that of the identity field in open string field theory [2].

Our work shows that this vertex, together with the open string vertex, generate a previously unsuspected decomposition of the moduli spaces of surfaces with boundaries and any number of punctures on the boundary and on the interior. The resulting Feynman rules have limitations: the closed strings must be on-shell, and, one cannot compute directly pure closed string processes. This is the price we pay for the extraordinarily simple decomposition of moduli space we achieve with the open-closed vertex. (Such difficulties might be generic in polynomial formulations). For a fully field-theoretical way of incorporating closed strings in open string theory we must use Ref. [1].

For closed strings we can generalize the usual string diagrams by imposing different length conditions on closed curves that correspond to external or internal strings (mathematically, on closed curves homotopic or nonhomotopic to punctures respectively). The resulting string diagrams interpolate between the standard covariant closed string diagrams [7, 8], the Strebel type string diagrams $[9,10]$ and a set of diagrams where the external closed strings appear as conical singularities of finite area. Here it is not clear that the family of string diagrams yield a family of string field theories.

The present results could give us some flexibility in dealing with the issue of background independence of string field theory. The reason is the following. After shifting by a classical solution, the resulting string field theory around the new background is defined in terms of a new set of effective vertices. It is possible, but difficult, to show that there is a field redefinition bringing the action back to the original form [11]. Perhaps the new effective vertices could be related to alternative vertices giving simply another decomposition of moduli space. At a more technical level, the reason why it is not manifest that a shift of the string field, via the three string vertex, yields a change in the BRST operator is that the latter is an insertion of a local operator, while the (covariant) vertex does not give a local insertion, it gives a smeared insertion, because all three strings in the vertex have finite length. The possibility of changing the length of a string consistently could help relate a local insertion to a smeared insertion. 
The open-closed theory for $l_{c}$ very small but finite may allow to treat the effects of changes in closed string backgrounds as local insertions in open string worldsheets. An alternative application might be in matrix models. Since the cubic action of open string field theory and its associated decomposition of moduli space are closely related to a matrix model with a background matrix [12], the action that will be discussed below, giving a new decomposition of moduli space could lead to a related matrix model integral. Finally, some physicists believe that the final form of string field theory should incorporate all possible ways of decomposing moduli space. Understanding how a family of decompositions (such as that presented here) could be put together into a single theory would be a first step in such program.

Before we begin, we should comment on the rigor of the presentation. We will prove that the string diagrams including closed strings are solutions of the minimal area problem, thus showing there cannot be overcounting. We will not, however, give an existence theorem for these quadratic differentials, so in principle, the Feynman rules could leave holes in moduli space. This seems very unlikely to be the case, because we will show, by analyzing recursion relations, that except for singular surfaces, the boundaries of the Feynman graphs contibuting to any amplitude always match.

The Open-Closed Vertex. Here we are looking for a string diagram for a disk containing one open and one closed string puncture. On the string diagram any nontrivial open curve should be longer than $\pi$, and the area ought to be minimal. If we had an infinite strip it would correspond to a disk with two open string punctures, thus the strip must be semiinfinite, and of width $\pi$, as shown in Fig. 1(a). Let us find out how to place the closed string puncture on this strip. Suppose it is located at $A$, namely at a point off the line cutting the strip in two equal pieces (shown with dots in the figure). This leads to a contradiction since there would be nontrivial open curves shorter than $\pi$. The puncture must therefore be on the middle of the strip. Suppose the puncture is at $B$ (on the middle line but away from the end of the semiinfinite strip). This is still problematic. It contradicts the saturation condition [7] which requires that through every point $Q$ on the surface there should be a nontrivial open curve of length $\pi^{*}$ There is, however, no such curve going through point $Q$ (Fig. 1(a)), the obvious

* If this is not the case, the value of the metric on a small neighborhood of $Q$ can be reduced without violating any length condition, and thus the area can be reduced. This contradicts the fact that the metric is supposed to be of minimal area. 
candidate, a vertical segment through $Q$ is homotopically trivial. This shows the puncture should be pushed all the way to $C$ (the end of the strip). There, however, it would lie on the boundary of the strip, which is not possible. We must therefore identify the $C D$ and $C D^{\prime}$ segments. The end result is the surface shown to the right (Fig. 1(b)).

The closed string is represented by the puncture at $C$, where we have a conical singularity of finite area. The surface is flat everywhere except at this point, where the curvature is positive and there is a defect angle of $\pi$. It corresponds to a first order pole of a quadratic differential. Let us explain this. The metric $\rho$ on the string diagram (recall $d l=\rho|d z|$ ) arises from a quadratic differential $\varphi=\phi(z) d z^{2}$ via the relation $\rho=|\phi|^{1 / 2}$. For our case, in the natural coordinate $w$, where the strip appears as shown in Fig. $2, \rho(w)=1$ and $\varphi(w)=d w^{2}$. The $w$ coordinate, centered on $C$, is mapped to a full neighborhood of the complex plane via $z=w^{2}$ (Fig. 2(b)). Using $\phi(z) d z^{2}=\phi(w) d w^{2}$, one readily finds that $\varphi(z)=d z^{2} / z$, hence the name first order pole of a quadratic differential. The horizontal trajectories of the quadratic differential are shown both in the $w$ and in the $z$ plane (for more details see [9]).

This vertex can define an off-shell amplitude. The strip defines canonical coordinates as far as the open string puncture is concerned. For the closed string puncture the $z$ coordinates can be defined to be the local coordinates. Namely, for any point $P$ sufficiently close to $C$ we define $z(P)=(w(P))^{2}$. This allows us to insert off-shell closed string states at $C$. The $z$ coordinates do not extend beyond the images of the vertical boundaries of the strip. The coordinates cannot be extended either beyond the image of a horizontal $w$ line where a curvature singularity appears. This difficulty becomes relevant for more complicated string diagrams.

Open-Open-Closed Amplitude. Analysis of this amplitude will now show that, in contrast with the case of light-cone field theory, or the open-closed covariant theory, there will be no need for an elementary interaction in order to cover fully the moduli space of the relevant Riemann surfaces. These surfaces are disks with one closed string puncture and two open string punctures in the boundary of the disk, and have one modular parameter, which can be chosen to be the angle $\theta$ shown in Fig. 3(a). Using our Feynman rules we obtain the string diagram shown to the right. The closed string, represented by the puncture at $P$, via the open-closed vertex turns instantaneously into an open string, which propagates for time $T$, and splits, via the symmetric open string vertex into open strings 1 and 2 . Note that 
large $T$ corresponds to small $\theta$, since effectively the open strings are becoming close to each other. When $T \rightarrow 0$ the string diagram turns into a completely flat semiinfinite strip with the closed string inserted on the middle of the strip (with no curvature singularity). This happens because at $T \rightarrow 0$, segments $O A$ and $O B$ get identified. This configuration corresponds to $\theta=\pi / 2$. Since we are dealing with orientable open strings, configurations with $\theta$ and $\pi-\theta$ are not equivalent. The diagram in Fig. 3(a) gave us the region $\theta \in[0, \pi / 2]$, the diagram in Fig. 3(b) gives us the region $\theta \in[\pi / 2, \pi]$. In the second diagram, when $T \rightarrow 0$, we find exactly the same configuration as we did in the previous case. It is clear we have covered completely moduli space, and we did not need an extra elementary interaction. As punctured surfaces with metrics the two string diagrams obtained in the limit as $T \rightarrow 0$ are identical.

Covering moduli space, however, is not sufficient in order to have a well defined off-shell amplitude. We do not seem to be able to get a consistent BRST invariant amplitude if we insist on having off-shell closed strings. The problem arises because we are unable to define the local coordinates around the closed string puncture smoothly over moduli space. For any specific surface, it is sufficient to specify coordinates in any nonvanishing neighborhood of the puncture. Note that, given the way we are defining coordinates around the closed string puncture, they cannot be extended beyond $|z|=T^{2}$, since the flat strip where the closed string puncture lies has a metric singularity at $w=i T$, the interaction point of the three open strings. As we let $T \rightarrow 0$, we eventually cannot define local coordinates around the closed string punctures. For any $T \neq 0$ we can define coordinates, but the limit as $T$ goes to zero does not exist. Indeed the closed string puncture, which sits on top of a first order pole of the quadratic differential collides with the first order zero at the interaction point. The result is a regular point, and we lose the ability to define coordinates smoothly. This discontinuity of local coordinates on the boundary of the two string diagrams implies that we cannot match the off-shell amplitudes, and prevents us from getting a BRST invariant amplitude.

Let us consider now the amplitude for scattering of three open strings and one closed string via a disk. We just check that the Feynman rules we have derived so far suggest that we cover fully moduli space. We do so by verifying that the boundaries of the Feynman graphs match. The Feynman rules are so simple, that there is just one type of graph structure involving two three-open-string vertices and one open-closed vertex. A particular assignment of the open strings is shown in Fig. 4 (a). The limits when either $T$ or $T^{\prime}$ go to infinity correspond to 
open string poles, and are therefore not considered to be boundaries of moduli space. We only need to consider the cases when $T$ or $T^{\prime}$ go to zero. When $T$ goes to zero the diagram matches with the diagram of Fig. 4(b). When $T^{\prime}$ goes to zero, it matches with the diagram of Fig. 4(c). Writing out all the relevant assignments of open strings, similar arguments show that all boundaries match. Instead of checking further examples, we will now use the geometrical recursion relations of the theory to argue systematically that further elementary interactions are not needed in order to cover moduli space.

Satisfying the Recursion Relations. We have now evidence that the Feynman rules of the following Lagrangian

$$
\mathcal{L}=\int\left(A Q A+\frac{2}{3} g A^{3}\right)+\hbar^{\frac{1}{2}} g \int A \Psi
$$

generate string diagrams that provide a single cover of moduli space. The first term in this lagrangian is the Witten open string field theory, with $A$ the open string field, and the second term is the open-closed interaction that we have discussed above. As explained in [1], for consistency of the loop expansion, this interaction appears at order $\hbar^{1 / 2}$. Note that there is no kinetic term for the closed string field $\Psi$, but the Feynman graphs will give rise to all required closed string poles. This action does not enable us to compute amplitudes involving surfaces without boundaries, namely, pure closed string processes.

The above lagrangian may not to be truly fundamental, since it fails to give BRST invariant off-shell amplitudes for procesess involving off-shell closed strings. It generates, however, all the relevant moduli spaces in an extremely simple way.

Since we expect covering of moduli space by the string diagrams generated by the Feynman rules, a geometrical consistency condition relating vertices must hold. This condition, first derived for closed strings in [14], was generalized to a theory including open strings in [1]. For each scattering amplitude, which involves a specific moduli space, there is a potential elementary vertex filling some region of that moduli space. The consistency condition demands that the surfaces in the boundary of the region of moduli space filled by this vertex should coincide with the appropriate string diagrams involving one propagator only, in the limit when the propagator collapses. The moduli space, or type of amplitude is specified by the genus $G$ of the surface, the number $N$ of closed string punctures, the number $B$ of boundary components, and the numbers $m_{i}, i=1 \cdots B$, which give the number of open string punctures in each 
boundary components. A particular vertex will be denoted by $\mathcal{V}_{B, M}^{G, N}$ (with $M=\sum m_{i}$ ). The dimension of a vertex will denote the dimension of the corresponding moduli space. Given that we only have open string propagators, the consistency condition is that given in Fig. 5, a subcase of the consistency condition given in [1], where we also had closed string propagators. (A discussion of geometrical consistency conditions can be found in [15].)

We want to see if it is consistent to have just a three open string vertex $\mathcal{V}_{1,3}^{0,0}$ and an openclosed vertex $\mathcal{V}_{1,1}^{0,1}$, and to set all others $\mathcal{V}_{B, M}^{G, N}$ to zero. There is no need to check an infinite number of vertices. The consistency conditions relate a vertex of dimension $d$, in the left hand side of the equation to either two vertices whose dimensions add up to $(d-1)$, or to a single vertex of dimension $(d-1)$. The two vertices we have correspond to $d=0$ and we claim that all other $d=0$ vertices vanish. If we can show that under this assumption no $d=1$ vertex is necessary, then it is consistent to set all higher dimensional vertices $(d \geq 2)$ equal to zero.

The dimension zero vertices we include correspond to the disk with three open string punctures and the disk with one open and one closed puncture (we do not include the disk with one or two open string punctures, nor the disk with one closed string puncture). Since the consistency condition is a consequence of correct covering of moduli space, in order to verify that the dimension one vertices can be set to zero we check that these moduli spaces are completely covered once we use the Feynman rules based on our dimension zero vertices.

The dimension one moduli spaces are: (a) the disk with four open string punctures, which is well covered in the usual open string theory [2], and therefore does not require the introduction of an elementary vertex; (b) the disk with one closed string and two open strings, which is also well covered, as we found above that no open-open-closed vertex was required; (c) the annulus with one open string puncture, which is the open string tadpole calculated with Witten's vertex, and which covers moduli space (but gives a singular Riemann surface in the right hand side of the geometrical equation, leading to regulatization problems), and finally, (d) the disk with two closed string punctures; this moduli space is well covered by the relevant string diagram, shown in Fig. 6 (this configuration also gives a singular term in the geometrical equation, corresponding to $T=0$ ). Since we have found complete covering, this shows that all dimension one vertices can be set to zero, and as a consequence all higher dimension vertices can be set to zero as well.

The consistency condition that we have verified is a necessary condition for getting a single 
cover of moduli space. Since it is not a sufficient condition, rigorously speaking, we have only obtained evidence that we have a single cover. The evidence will be furthered now by showing that the Feynman rules build minimal area string diagrams. A complete proof would require an analysis similar to that of [3], namely establishing an existence and uniqueness theorem for quadratic differentials having first and second order poles. The methods of [3] are likely to be applicable, but we will not try to construct such a proof here.

Minimal Area Property of the String Diagrams. It was explained precisely in [16] why the minimal area property guarantees that no surface is produced more than once by the Feynman rules. The basic idea is that two different Feynman diagrams must produce different metrics, both of minimal area. But for a given surface there is just one metric of minimal area, thus the two Feynman diagrams must be building different Riemann surfaces.

The minimal area property for the present case follows from the following two facts: (a) on every strip the open curves extending from one boundary to the other are nontrivial, and (b) any nontrivial open curve is longer or equal to $\pi$. Property (a) holds because if we double the string diagram we get a meromorphic Jenkins-Strebel quadratic differential (with both first order and second order poles). In this surface the double of the open curves lying on a strip are curves homotopic to core curves of the ring domains. Since core curves are nontrivial, the closed curves in question must be nontrivial. But a nontrivial closed curve built by doubling must arise from a nontrivial open curve [16]. Property (b) holds because any nontrivial open curve must cross the line that goes along the middle of all the strips in the string diagram. Therefore, the metric satisfies the necessary length conditions. The area is minimal because it is minimal under the weaker condition that the open curves in each of the strips be greater or equal to $\pi$. This is so because it is made of flat strips.

Interpolating String Field Theories. We have seen that it is possible to have closed string punctures and to have length conditions on open curves only. This is the limit case of a more general problem that imposes length conditions on both open and closed curves. We can pose the following problem for open-closed string theory: the string diagrams are defined by the metric of minimal area under the condition that all nontrivial Jordan open curves be longer or equal to $l_{o}$ and all nontrivial Jordan closed curves be longer or equal to $l_{c}$. As long as $l_{o} \neq 0$ we can scale the string diagrams to $l_{o}=\pi$, and we get a one parameter family of string 
diagrams. They interpolate from the open-closed field theory [1], which has $l_{c}=2 \pi$, to the theory studied here, which corresponds to $l_{c}=0$. A sequence of open closed string diagrams is shown in Fig. 7. As long as we keep $l_{c} \neq 0$ the string diagrams may be reconstructed using vertices and propagators, in a way similar to that of [1]. Thus we get a a one-parameter family of consistent off-shell string field theories. Some of the minimal area metrics will not arise from quadratic differentials. As $l_{c} \rightarrow 0$ one can verify that the higher open-closed vertices fill progressively smaller regions of moduli space. For $l_{c}=0$, only the open-closed vertex is relevant, and the off-shell property is lost. We then get simple string diagrams arising from quadratic differentials, as we showed earlier.

For closed strings one can also generalize the string diagrams. Since modular transformations do not mix Jordan closed curves homotopic to punctures with Jordan closed curves that are not homotopic to a puncture we can put different length conditions for the two types of curves. We obtain a one parameter family of generalized closed string diagrams by requiring that the area be minimal under the conditions that nontrivial Jordan closed curves not homotopic to punctures be longer or equal to $l_{c}$ and those homotopic to punctures longer or equal to $l_{p}$. The standard closed string field theory corresponds to $l_{c}=l_{p}=2 \pi$. Another familiar case is $l_{c}=0$ and $l_{p}=2 \pi$; it corresponds to the Strebel construction of every surface as a contact interaction [10]. Thus the one parameter family of string diagrams with $l_{p}=2 \pi$ and $l_{c}$ variable interpolate from the standard closed string diagrams into the Strebel construction of every surface as a polyhedron.

The string diagrams where both $l_{c}$ and $l_{p}$ are different from each other, and different from zero do not seem to arise from naive Feynman rules of string field theory. This is the case because putting different length conditions for curves surrounding punctures and for other curves is incompatible with naive sewing. If we sew together two elementary vertices we get an internal cylinder with circumference $l_{p}$. If $l_{p}<l_{c}$, this violates the length conditions, and if $l_{p}>l_{c}$, it is clear that for a sufficiently long cylinder, the saturation condition [7] is violated, and the metric could not be of minimal area (in the middle of the tube we could not find a nontrivial curve of length $l_{c}$ ). In these string diagrams all degenerations happen nicely via long tubes, but we have no off-shell factorization.

The case $l_{c}=2 \pi$ and $l_{p}=0$, corresponds to no conditions on curves homotopic to the punctures, thus the punctures appear typically as conical singularities. Note, however, that 
in contrast with the case of open strings, we do not expect off-shell problems here, since the closed string punctures cannot collide (any nontrivial curve surrounding two punctures must be longer or equal to $2 \pi$ ). It may be interesting to elucidate the structure of these string diagrams.

We hope to have illustrated here the power of minimal area methods. All decompositions of moduli space used in covariant string field theory arise from minimal area metrics. We have found here that we can easily generate families of decompositions that interpolate between various known and new decompositions. In one case we get a family of string field theories. A possible utility of our results would be an enlarged framework for string field theory where background independence would be easier to understand.

Acknowledgements: I am grateful to R. Dijkgraaf, T. Kugo and E. Witten for discussions.

\section{REFERENCES}

1. B. Zwiebach, "Quantum Open String Theory with Manifest Closed String Factorization", Phys. Lett. B256 (1991) 22.

2. E. Witten, "Noncommutative Geometry and String Field Theory", Nucl. Phys. B268 (1986) 253.

3. B. Zwiebach, "A proof that Witten's open string theory gives a single cover of moduli space", Comm. Math. Phys.

4. J. A. Shapiro and C. B. Thorn, Phys. Lett. B194 (1987) 43.

5. A. Strominger, Phys. Rev. Lett. 58 (1987) 58.

6. I. A. Batalin and G. A. Vilkovisky, Phys. Rev. D28 (1983) 2567.

7. B. Zwiebach, Mod. Phys. Lett. A5 (1990) 2753.

8. B. Zwiebach, Comm. Math. Phys. 136 (1991) 83.

9. K. Strebel, Quadratic Differentials, Springer-Verlag, Berlin, 1984.

10. M. Saadi and B. Zwiebach, Ann. Phys. 192 (1989) 213.

11. A. Sen, "On the Background Independence of String Field Theory. III. Field Redefinitions", Tata Institute Preprint, TIFR-TH-92/03, January 1992. 
12. M. Kontsevich, "Intersection Theory in the Moduli Space of Curves and the Matrix Airy Function", Max-Planck-Institut preprint, MPI/91-77.

13. C. B. Thorn, "String field theory", Phys. Rep. 174 (1989) 1.

14. H. Sonoda and B. Zwiebach, Nucl. Phys. B331 (1990) 592.

15. B. Zwiebach, "Recursion Relations in Closed String Field Theory", Proceedings of the "Strings 90" Superstring Workshop at Texas A\& M. Eds. R. Arnowitt, et. al. (World Scientific, 1991) pp.266-275.

16. B. Zwiebach, "Minimal area problems and quantum open strings", Comm. Math. Phys. 141 (1991) 577. 


\section{Figure Captions}

Figure (1). (a) Finding out where to place the closed string puncture on the strip. Positions $A$ and $B$ are not possible. The closed string must be placed at $C$ and the segments $C D$ and $C D^{\prime}$ have to be identified. (b) The resulting vertex geometry.

Figure (2). (a) The open-closed vertex shown in the $w$-plane, where it appears as a semiinfinite strip with the closed string puncture at $C$, and with $C D$ and $C D^{\prime}$ identified. (b) The mapping $z=w^{2}$ maps the strip into the $z$ plane. Here the quadratic differential takes the form $d z^{2} / z$ corresponding to a first order pole. The horizontal trajectories are shown both in the $w$ and in the $z$ plane.

Figure (3). In open-open-closed scattering the relevant moduli space can be parametrized by the angle $2 \theta$ separating the open string punctures in a unit disc where the closed string puncture is at the center. (a) $\theta \in[0, \pi / 2]$; the string diagram having an open string propagator, and the limit as this propagator collapses. (b) $\theta \in[\pi / 2, \pi]$. It is clear that in the limit when the two propagators collapse the resulting surfaces (with metrics) are identical.

Figure (4). String diagrams for scattering of three open strings and one closed string via a disk. Configurations match as the open string propagators collapse.

Figure (5). The geometrical consistency conditions for elementary vertices in the extended covariant open string theory. The boundary of an elementary interaction must coincide with a configuration with a single open string propagator, in the limit when this propagator collapses. The wiggly lines correspond to external closed strings, and the other lines represent open strings. The heavy dots represent boundary components.

Figure (6). The string diagram corresponding to the scattering of two closed strings via a disk. The corresponding moduli space is one dimensional and is generated completely by the string diagram shown above. When the open string propagator collapses the two closed string punctures collide giving a singular limit.

Figure (7). We show how the open-closed vertex varies as we change the length condition on the closed curves. We show four cases, corresponding to $l_{c}>l_{o}, l_{c}=l_{o}, l_{c}<l_{o}$, and $l_{c}=0$. 